\title{
Formalin fixation and patterns of antineutrophil cytoplasmic antibodies
}

\author{
G P Spickett, V Broomhead
}

\begin{abstract}
Recent publications have suggested that fixing neutrophils in formalin is a useful adjunct to the differentiation of antinuclear antibodies from perinuclear antineutrophil cytoplasmic antibodies. In this small comparative study of 30 sera the use of an additional slide of formalin fixed neutrophils did not add any useful information and may be confusing as the results were not reproducible.

( Clin Pathol 1995;48:89-90)
\end{abstract}

Keywords: Antineutrophil cytoplasmic antibodies, vasculitis.

Antineutrophil cytoplasmic antibodies (ANCA) are useful for identifying patients with systemic vasculitis. A serendipitous discovery that ethanol fixation of human neutrophils leads to the redistribution of target antigens in the cytoplasm has permitted the recognition of two distinct patterns of immunofluorescence. The cytoplasmic ANCA (c-ANCA) pattern is most closely associated with Wegener's granulomatosis and with microscopic polyarteritis, while the perinuclear ( $p$-ANCA) pattern is seen in a broad range of systemic vasculitides, especially those involving the kidney. The target antigen of c-ANCA has been identified as proteinase-3, while p-ANCA recognise a range of neutrophil proteins, including myeloperoxidase, lactoferrin, and elastase. ${ }^{1}$

Solid phase assays for the detection of these antibodies are being introduced, but have led to significant standardisation problems. The standard method for the detection of ANCA is immunofluorescence. This was established at the first international workshop on ANCA in 1988, and has been reconfirmed at subsequent workshops and by the EEC/ BCR Group for ANCA Assay Standardisation. The first workshop in 1988 defined a standard procedure for carrying out the immunofluorescence assay, using ethanol fixed neutrophils as the substrate. ${ }^{2}$ This methodology has subsequently been used by the EEC/BCR group. ${ }^{3}$ Recently, the Association of Clinical Pathologists prepared a broadsheet discussing the methodology of ANCA detection. ${ }^{4}$ This broadsheet introduces an additional step, using neutrophils fixed in formalin-acetone followed by absolute ethanol in addition to ethanol fixed neutrophils, to distinguish p-ANCA from granulocyte specific antinuclear antibodies (GS-ANA). This additional step has not been validated in the international workshops, although it has been used before. A number of groups, including ours, have tried this technique in the past and found it to be unsatisfactory. The problems associated with it have been discussed informally at the last three international workshops but no significant comparisons have been published. Lee et $a{ }^{5}$ have claimed success using formaldehyde vapour to convert a $\mathrm{p}$-ANCA, resulting from antimyeloperoxidase antibodies, to a cANCA pattern. The timing of exposure of the substrate to the vapour was critical. These authors commented that liquid formalin and formalin-acetone, as recommended by Lock, ${ }^{4}$ gave inconsistent results.

As our own experience of formalin fixation was similar to that described by Lee $e t a l,{ }^{5}$ we undertook a comparison of staining of human neutrophils and rat liver for differentiating between ANA, GS-ANA, and p-ANCA.

\section{Methods}

Thirty sera which had previously been received for detection of ANCA or ANA were used. Antinuclear antibodies were detected by titration on multiblock cryostat sections, comprising rat liver, mouse kidney and stomach. Bound IgG was detected using antihuman IgG fluorescein isothiocyanate (FITC) (Dako, High Wycombe, UK) at a dilution of 1 in 40 . The starting serum dilution for this experiment was 1 in 10 , but an ANA titre of less than 1 in 40 would not be considered clinically important.

Antineutrophil cytoplasmic antibodies were detected on ethanol fixed human neutrophils, as described by Wiik. ${ }^{2}$ The same starting dilution and FITC conjugate were used. Two batches of human neutrophils from the same donor as the ethanol fixed neutrophils were prepared using formalinacetone fixation followed by absolute ethanol, as described by Lock. ${ }^{4}$

The initial reading of ethanol fixed and formalin fixed slides was carried out in an "open" manner. The samples were then blinded and re-run on the first batch of formalin fixed slides. A new batch of formalin fixed slides was then prepared and the samples re-blinded and run. All slides were read by an experienced MLSO (VB).

The standard laboratory reporting protocol requires that any p-ANCA positive samples on ethanol fixed neutrophils are screened for ANA on rat liver. The specimen is only reported as p-ANCA positive if the titre of 
Detection of antineutrophil cytoplasmic antibodies using immunofluorescence

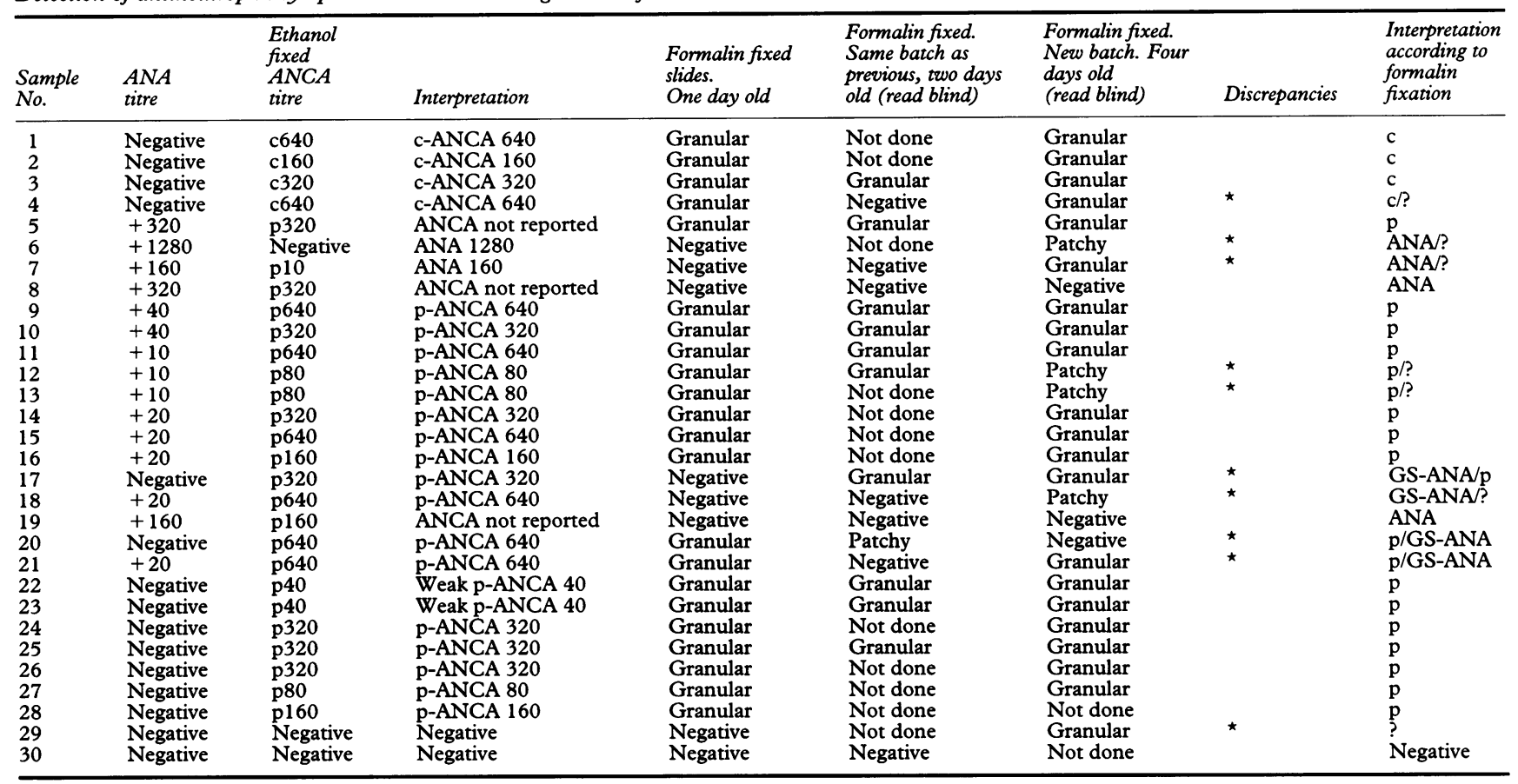

ANA = antinuclear antibodies; $A N C A=$ antineutrophil cytoplasmic antibodies; GS-ANA = granulocyte specific ANA; p-ANCA = perinuclear ANCA; c-ANCA = cytoplasmic ANCA; Granular = coarse cytoplasmic granular staining.

staining on neutrophils is at least fourfold greater than that seen on rat liver. If this condition is not fulfilled the sample is reported as "ANA present-p-ANCA unreportable". Rat liver and human neutrophils cannot be considered equivalent substrates for ANA, and not all samples positive for ANA on one substrate will have a positive result on the alternative substrate. This rule, however, prevents over-interpretation of results obtained on neutrophil immunofluorescence.

\section{Results}

The results obtained on the 30 samples are presented in the table. Ten samples gave discrepant results when the results of the three runs on formalin fixed cells were compared. Samples 6 and 7, both with very strong ANA on rat liver, had negative cytoplasmic fluorescence on the first two runs but had granular cytoplasmic staining on the third run, which, if seen alone, might have suggested the presence of p-ANCA. For sample 4, which was known to be strongly c-ANCA positive, one of the formalin fixed neutrophil runs produced negative staining; conversely, sample 29, which was selected as a negative control, gave positive granular staining on one of the formalin fixed runs. The remaining discrepant results related to p-ANCA positive samples, with one or more of the formalin fixed slides giving negative or patchy cytoplasmic fluorescence.

\section{Discussion}

It appears from this study that patchy staining was more likely to be seen on stored than on fresh formalin fixed slides. This was not investigated specifically, but may contribute further to the unreliability of this method. A need to prepare fresh neutrophils on a daily basis would add substantially to the cost of the assay.

These results confirm that formalin fixation does not contribute substantially to the identification of p-ANCA staining. Of particular concern was the lack of reproducibility of the staining patterns on formalin fixed neutrophils. Under these circumstances, we feel that this method should not be used routinely at present. While we accept that our comparison of neutrophil staining against rat liver staining may not permit definitive differentiation between ANA, GS-ANA, and p-ANCA, it does prevent over-interpretation of the ANCA assay. The use of formalin fixation, however, appears to be less accurate still. Diagnostic testing for ANCA using immunofluorescence should be restricted to methods that have been validated by the international workshops ${ }^{23}$ until formalin fixation has been examined in more detail.

1 Gross WL, Schmitt WH, Csernok E. ANCA and associated diseases: immunodiagnostic and pathogenetic aspects. Clin Exp Immunol 1993;91:1-12.

2 Wiik A. Delineation of a standard procedure for indirect immunofluorescence detection of ANCA. Acta Pathol Microbiol Immunol Scand 1989;97(Suppl 6): 12-13.

3 Hagen EC, Andrassy K, Chernok E, Daha MR, Gaskin $\mathrm{G}$, Gross $\mathrm{W}$, et al. The value of indirect immunofluorescence and solid phase techniques for ANCA detection. A report on the first phase of an international co-operaA report on the first phase of an international co-opera-
tive study on the standardisation of ANCA assays. $f$ tive study on the standardisatio

4 Lock RJ. Detection of autoantibodies to neutrophil cytoplasmic antigens. F Clin Pathol 1994;47:4-8.

5 Lee SS, Lawton JWM, Chak W. Distinction between antinuclear antibody and p-ANCA. F Clin Pathol 1991;44: 962-3. 